DOI: 10.20472/IAC.2017.029.002

MOTASEM ABU DAWAS

Irbid National University, Jordan

\title{
A NEW METHOD TO BUILD CAD SYSTEM ARCHITECTURE BASED ON A UNIFORM PROCEDURAL SYSTEM OF OPERATING COMPONENTS
}

\begin{abstract}
:
This paper deals with one of the important issues of Automated Design (AD) which is the formation and periodic reconfiguration of Computer Aided Design (CAD). The paper proposes a method to building CAD system architecture that based on a uniform procedural system of operating components, which reflect the specifics of concrete CAD applications and can improve the reliability and validity of the choice of system architecture. Implemented structural and functional decomposition model of operating components and the generic object of design, analysis of typical design procedures. Proposed use a modified system of logical rules of transformations of model project descriptions in the form of basic operating components of the process. The system of logical rules based on the structural and functional decomposition of standard operating components of CAD and generic object of design.
\end{abstract}

\section{Keywords:}

Computer Aided Design

JEL Classification: C63 